\title{
ERRATUM
}

\section{Common SNPs in LEP and LEPR associated with birth weight and type 2 diabetes-related metabolic risk factors in twins}

NY Souren, AD Paulussen, A Steyls, RJ Loos, AP Stassen, M Gielen, HJ Smeets, G Beunen, R Fagard, C Derom, R Vlietinck, JP Geraedts and MP Zeegers

International Journal of Obesity (2008) 32, 1335-1336; doi:10.1038/ijo.2008.93

Correction to: International Journal of Obesity advance online publication, 20 May 2008; doi:10.1038/ijo.2008.68

Owing to a typesetting error in Tables 1 and 2 of the above paper some cells and the background of some text in the legend are gray, while they should have been white.

The correct tables are reproduced below.

The typesetters would like to apologize for this error.

Table 1 Phenotypic characteristics of MZ and DZ twins by gender

\begin{tabular}{|c|c|c|c|c|c|c|}
\hline \multirow[t]{2}{*}{ Characteristic } & \multicolumn{3}{|c|}{$M Z$} & \multicolumn{3}{|c|}{$D Z$} \\
\hline & Men & Women & P-value & Men & Women & P-value \\
\hline$n$ & 179 & 217 & & 107 & 125 & \\
\hline Birth weight (g) & $2540 \pm 443$ & $2456 \pm 488$ & 0.11 & $2690 \pm 446$ & $2572 \pm 474$ & 0.002 \\
\hline Gestational age (weeks) ${ }^{a}$ & $36.9 \pm 2.2$ & $36.8 \pm 2.6$ & 0.98 & $37.4 \pm 2.2$ & $37.5 \pm 2.3$ & 0.82 \\
\hline Age (years) ${ }^{a}$ & $25.0 \pm 4.6$ & $24.8 \pm 4.7$ & 0.68 & $25.5 \pm 4.7$ & $25.6 \pm 4.6$ & 0.88 \\
\hline Body height $(\mathrm{cm})$ & $178.0 \pm 5.9$ & $165.3 \pm 6.5$ & $<0.0001$ & $178.5 \pm 6.7$ & $166.5 \pm 6.5$ & $<0.0001$ \\
\hline Body mass (kg) & $69.3 \pm 9.1$ & $60.2 \pm 9.9$ & $<0.0001$ & $70.1 \pm 10.1$ & $60.7 \pm 10.0$ & $<0.0001$ \\
\hline BMI $\left(\mathrm{kg} / \mathrm{m}^{2}\right)^{\mathrm{b}}$ & $21.7 \pm 1.1$ & $21.8 \pm 1.2$ & 0.78 & $21.9 \pm 1.1$ & $21.7 \pm 1.1$ & 0.84 \\
\hline Fat mass $(\mathrm{kg})^{\mathrm{b}}$ & $11.3 \pm 1.5$ & $16.7 \pm 1.3$ & $<0.0001$ & $12.2 \pm 1.5$ & $16.6 \pm 1.3$ & $<0.0001$ \\
\hline Lean body mass (kg) & $57.0 \pm 6.2$ & $42.8 \pm 5.2$ & $<0.0001$ & $57.0 \pm 6.5$ & $43.5 \pm 5.9$ & $<0.0001$ \\
\hline $\mathrm{S} 4 \mathrm{SF}(\mathrm{mm})^{\mathrm{b}}$ & $34.9 \pm 1.5$ & $56.2 \pm 1.4$ & $<0.0001$ & $34.2 \pm 1.5$ & $52.2 \pm 1.4$ & $<0.0001$ \\
\hline WHR (\%) & $82.9 \pm 5.3$ & $73.2 \pm 4.5$ & $<0.0001$ & $82.6 \pm 5.1$ & $72.3 \pm 4.2$ & $<0.0001$ \\
\hline IGFBP-1 ( $\left.\mathrm{ng} \mathrm{ml}^{-1}\right)^{\mathrm{b}}$ & $10.9 \pm 1.8$ & $16.3 \pm 2.0$ & $<0.0001$ & $12.2 \pm 1.8$ & $19.1 \pm 2.3$ & $<0.0001$ \\
\hline Fasting insulin $\left(\mathrm{pmoll}^{-1}\right)^{\mathrm{b}}$ & $32.5 \pm 1.5$ & $37.8 \pm 1.6$ & 0.005 & $31.6 \pm 1.6$ & $40.6 \pm 1.5$ & $<0.0001$ \\
\hline Fasting glucose $\left(\mathrm{mmoll}^{-1}\right)$ & $5.0 \pm 0.4$ & $4.6 \pm 0.4$ & $<0.0001$ & $4.8 \pm 0.4$ & $4.6 \pm 0.4$ & $<0.0001$ \\
\hline Leptin $\left(\mathrm{ng} \mathrm{ml} \mathrm{m}^{-1}\right)^{\mathrm{b}}$ & $1.6 \pm 3.1$ & $11.8 \pm 2.1$ & $<0.0001$ & $1.6 \pm 2.8$ & $11.4 \pm 1.9$ & $<0.0001$ \\
\hline Total cholesterol $\left(\mathrm{mmoll}^{-1}\right)$ & $4.8 \pm 1.0$ & $5.1 \pm 0.8$ & 0.003 & $5.0 \pm 1.1$ & $5.2 \pm 1.0$ & 0.08 \\
\hline LDL cholesterol $\left(\mathrm{mmoll}^{-1}\right)$ & $3.0 \pm 0.9$ & $2.9 \pm 0.8$ & 0.43 & $3.1 \pm 1.0$ & $2.9 \pm 0.8$ & 0.03 \\
\hline HDL cholesterol $\left(\mathrm{mmoll}^{-1}\right)$ & $1.4 \pm 0.3$ & $1.8 \pm 0.4$ & $<0.0001$ & $1.4 \pm 0.4$ & $1.9 \pm 0.4$ & $<0.0001$ \\
\hline Triacylglycerol $\left(\mathrm{mmoll}^{-1}\right)^{\mathrm{b}}$ & $0.8 \pm 1.5$ & $0.9 \pm 1.5$ & 0.04 & $0.9 \pm 1.5$ & $0.9 \pm 1.5$ & 0.81 \\
\hline $\operatorname{NEFA}\left(\mathrm{mmoll}^{-1}\right)$ & $0.5 \pm 0.2$ & $0.7 \pm 0.2$ & $<0.0001$ & $0.5 \pm 0.2$ & $0.7 \pm 0.2$ & $<0.0001$ \\
\hline
\end{tabular}

Abbreviations: BMI, body mass index; DZ, dizygotic; HDL, high-density lipoprotein; LDL, low-density lipoprotein; WHR, waist-to-hip ratio; IGFBP1, insulin-like growth factor protein 1; MZ, monozygotic; n, number of individuals; NEFA, non-esterified fatty acids; S4SF, sum of four skinfolds; WHR, waist-to-hip ratio. Data are expressed as mean \pm s.d. ${ }^{a} P$-value calculated using standard linear regression, because convergence criteria could not be met in the random intercept model.

${ }^{b}$ Geometric mean \pm s.d. 
Table 2 Significant associations between the SNPs in the LEPR and the LEP gene and the metabolic risk factors for type 2 diabetes; adjusted for significant covariates

\begin{tabular}{|c|c|c|c|c|c|c|c|c|}
\hline Gene (SNP) & Trait $^{\mathrm{a}}$ & & Genotype & & $\mathrm{P}_{G}$ & $\mathrm{P}_{A}$ & $P_{D}$ & $\mathrm{P}_{R}$ \\
\hline \multirow[t]{3}{*}{ LEPR (K109R) } & & KK & KR & $\mathrm{RR}$ & & & & \\
\hline & $n\left(n_{\mathrm{MZ}} / n_{\mathrm{DZ}}\right)^{\mathrm{b}}$ & $354(240 / 114)$ & $194(116 / 78)$ & $46(30 / 16)$ & & & & \\
\hline & Birth weight $^{c}$ & $2511(2465-2557)$ & $2575(2516-2635)$ & $2726(2606-2845)$ & 0.003 & 0.001 & 0.01 & 0.003 \\
\hline \multirow[t]{3}{*}{ LEPR (Q223R) } & & QQ & QR & $\mathrm{RR}$ & & & & \\
\hline & $n\left(n_{\mathrm{MZ}} / n_{\mathrm{DZ}}\right)^{\mathrm{b}}$ & $189(126 / 63)$ & $295(185 / 110)$ & $98(66 / 32)$ & & & & \\
\hline & Birth weight ${ }^{d}$ & $2492(2431-2554)$ & 2545 (2495-2595) & $2655(2571-2740)$ & 0.009 & 0.003 & 0.04 & 0.006 \\
\hline \multirow[t]{3}{*}{$\operatorname{LEP}(19 \mathrm{G}>\mathrm{A})$} & & GG & GA & AA & & & & \\
\hline & $n\left(n_{\mathrm{MZ}} / n_{\mathrm{DZ}}\right)^{\mathrm{b}}$ & $216(131 / 85)$ & $265(181 / 84)$ & $82(53 / 29)$ & & & & \\
\hline & $\mathrm{HDL}$ cholesterol $\mathrm{d}^{\mathrm{d}}$ & $1.59(1.53-1.65)$ & $1.64(1.59-1.69)$ & $1.49(1.40-1.58)$ & 0.02 & 0.35 & 0.64 & 0.01 \\
\hline
\end{tabular}

Abbreviations: $\mathrm{HDL}$, high-density lipoprotein; $n$, total number of individuals; $n_{\mathrm{DZ}}$, number of $\mathrm{DZ}$ individuals; $n_{\mathrm{MZ}}$, number of $\mathrm{MZ}$ individuals; $P_{\mathrm{A}}, P$-value additive model; $P_{\mathrm{D}}, P$-value dominant model; $P_{\mathrm{G}}, P$-value general association model; $P_{\mathrm{R}}, P$-value recessive model; SNP, single nucleotide polymorphism. Data are expressed as least squares mean $(95 \% \mathrm{Cl})$. The lowest $P$-value is shown in boldface type. See also Supplementary Tables 3,4 and 5 for the means $(95 \% \mathrm{Cl}$ ) per genotype group and the mode of inheritance (additive, dominant and recessive) of the associations that were considered nonsignificant. ${ }^{2}$ The units of the phenotypic characteristics are presented in Table 1. ${ }^{\mathrm{b}}$ Total number of individuals differ from the number reported in Table 1 because of missing values for the genotype, dependent variable or

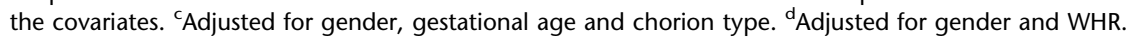

